\title{
Search for new physics in the B-sector at the LHC
}

\author{
Johannes Albrecht \\ Physikalisches Institut \\ Philosophenweg 12, 69120 Heidelberg \\ Germany
}

\begin{abstract}
A set of key measurements of $B$ decays which have the potential to uncover New Physics at the LHC is discussed. Together with the general purpose detectors ATLAS and CMS, the LHCb detector, which is devoted to $B$ physics, can study these effects precisely. Due to the large $b \bar{b}$ cross section at $14 \mathrm{TeV}, \mathrm{LHC} b$ has access to $10^{12} B$ meson decays per year. This allows significant measurements of even very rare $B$ decays and, in particular, the precision study of the $B_{s}$ system.
\end{abstract}

\section{Introduction}

The Large Hadron Collider (LHC) at CERN is a proton-proton collider which will operate at a center of mass energy of $14 \mathrm{TeV}$. Three of the LHC experiments search for New Physics signatures in the flavor sector: The ATLAS and CMS experiments study $B$ meson decays in parallel to their main focus on direct searches for New Physics and the Higgs boson. In contrast to that, the $\mathrm{LHC} b$ experiment is designed and optimized to measure precisely the $B$ meson properties. Therefore, this article puts the main focus on the expected LHCb measurements and, where available, compare them with the predictions from ATLAS and CMS.

To unambiguously assign long lived particles to their primary vertex, events with a single proton-proton interaction are preferred. $\mathrm{LHC} b$ therefore plans to adjust the beam luminosity to an average value of $2 \times 10^{32} \mathrm{~cm}^{-2} \mathrm{~s}^{-1}$. In contrast to that, the ATLAS and CMS experiments plan to run at the maximal LHC luminosity of $10^{34} \mathrm{~cm}^{-2} \mathrm{~s}^{-1}$.

The unprecedented quantity of $b$-hadrons that will be produced at the LHC allow stringent tests of the Standard Model and of possible New Physics scenarios. Indirect effects studied in $b$ hadron decays provide complementary information to that from direct searches and allow to probe the flavor structure of possible New Physics. One of the early measurements of major interest will be the determination of the $B_{s}$ mixing phase $\Phi_{s}$ from the decay $B_{s} \rightarrow J / \psi \phi$. Data with a few months of LHC running will allow to cross check the deviation from the Standard Model prediction currently indicated by the two Tevatron experiments [2]. Flavor changing neutral current decays such as $B_{s} \rightarrow \mu^{+} \mu^{-}$and $B_{d} \rightarrow K^{\star} \mu^{+} \mu^{-}$are very sensitive to different extensions of the Standard Model. In case no signatures of New Physics would be observed, possible New Physics scenarios will be significantly constrained. The measurement of the direct $\mathrm{CP}$ asymmetry in $B \rightarrow D K$ tree-level decays will result in a precise determination of the CKM angle $\gamma$, which in combination with indirect measurements can probe possible New Physics contributions.

\section{Measurement of the $B_{s}$ mixing phase}

The interference between $B_{s} \rightarrow J / \psi \phi$ decays with and without a $B_{s}-\bar{B}_{s}$ oscillation gives rise to the $C P$ violating asymmetry proportional to $\sin \Phi_{s}$. In the Standard Model, when 
penguin contributions to the decay amplitude are neglected, this phase is predicted to be

$$
\Phi_{s}=-2 \beta_{s}=(-0.0368 \pm 0.0017) \mathrm{rad},
$$

where $\beta_{s}=\arg \left(\left(V_{t s} V_{t b}^{*}\right) /\left(V_{c s} V_{c b}^{*}\right)\right)$. New Physics could significantly modify this by contributing to the $B_{s}-\bar{B}_{s}$ mixing diagram.

The final state $J / \psi \phi$ consists of two vector mesons, hence its $C P$-eigenvalue depends on the relative angular momentum of the two mesons. An angular analysis allows to disentangle on a statistical basis the $C P$-odd and $C P$-even components. The time dependent $C P$ asymmetry $A_{C P}(t)$ is given by

$$
A_{C P}(t)=\frac{\eta_{f} \sin \Phi_{s} \sin \left(\Delta m_{s} t\right)}{\cosh \left(\Delta \Gamma_{s} t / 2\right)-\eta_{f} \cos \Phi_{s} \sinh \left(\Delta \Gamma_{s} t / 2\right)},
$$

where $\Delta m_{s}$ and $\Delta \Gamma_{s}$ are the differences in mass and width of the two $B_{s}$ mass eigenstates and $\eta_{f}= \pm 1$ is the $C P$ eigenvalue of the final state.

$\mathrm{CDF}$ and D $\varnothing$ recently published measurements of the time dependent $C P$ asymmetry for the channel $B_{s} \rightarrow J / \psi \phi$, which use 1.3 and $2.8 \mathrm{fb}^{-1}$ of data respectively [2]. The HFAG combination [3] results in the following value of the weak mixing phase $\Phi_{s}$,

$$
\Phi_{s}=\left[\left(-0.77_{-0.37}^{+0.29}\right) \text { or }\left(-2.36_{-0.29}^{+0.37}\right)\right] \mathrm{rad} .
$$

This result shows a possible deviation from the Standard Model prediction. An update by CDF [4] using $2.8 \mathrm{fb}^{-1}$ further degrades the consistency with the SM.

For the decay $B_{s} \rightarrow J / \psi \phi$, the $\mathrm{LHC} b$ experiment expects a proper time resolution of $38 \mathrm{ps}$, a $B_{s}$ mass resolution of $16 \mathrm{MeV}$ and an effective tagging power $\left(\epsilon D^{2}\right)$ of $6.2 \%$. With a total efficiency of $2.6 \%$ including trigger, the simulation predicts a yield of 117000 selected signal events per $2 f^{-1}$ with a background-to-signal ratio of about 0.5 [5]. The $\Phi_{s}$ sensitivity has been estimated to be

$$
\begin{array}{cl}
\mathcal{L}=0.5 f b^{-1} & : \quad \sigma\left(\Phi_{s}\right)=0.060 \pm 0.005 \\
\mathcal{L}=2 f b^{-1} & : \quad \sigma\left(\Phi_{s}\right)=0.030 \pm 0.002
\end{array}
$$

where only statistical errors are given. Systematic effects due to proper time and angular resolutions, angular acceptance and flavor tagging have been studied and found to be smaller than the statistical uncertainty expected for $2 \mathrm{fb}^{-1}$. Figure 1 shows the expected $\mathrm{LHC} b$ statistical uncertainty for $\Phi_{s}=-2 \beta_{s}$ versus the integrated luminosity $\mathcal{L}_{\text {int }}$. The red line is an extrapolation from the above two values. The blue lines show the uncertainties related to the $b \bar{b}$ crosssection and the visible branching ratio of

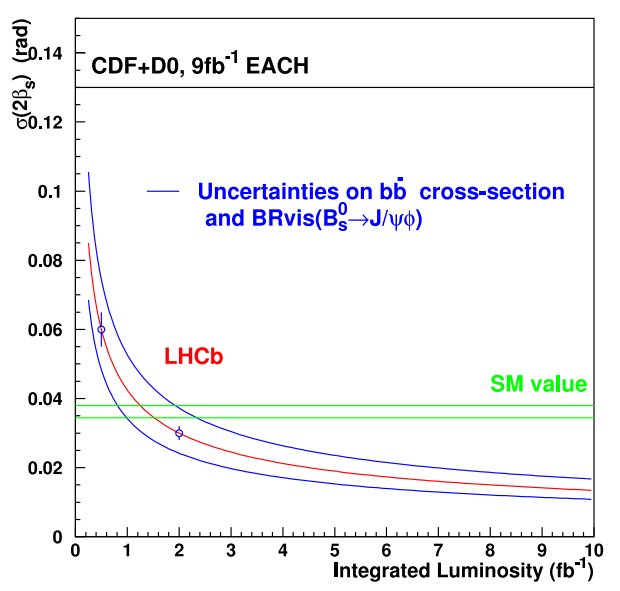

Figure 1: Expected LHCb sensitivity on $\Phi_{s}=$ $-2 \beta_{s}$ as a function of the integrated luminosity. The Standard Model prediction and the Tevatron expectation for 2010 are indicated. $B_{s} \rightarrow J / \psi(\mu \mu) \phi(K K)$. The black vertical

line is the combined $\mathrm{CDF} / \mathrm{D} \varnothing$ uncertainty in 2008 scaled to an integrated luminosity of $2 \times 9 \mathrm{fb}^{-1}$, as expected for the two Tevatron experiments by 2010 . It should be noted that $\mathrm{LHC} b$ is able to perform a competitive measurement of $\Phi_{s}$ already with the very first data. 


\section{Search for New Physics in flavor changing neutral current processes}

\section{$3.1 \quad B_{s} \rightarrow \mu^{+} \mu^{-}$}

The very rare decays $B_{d, s}^{0} \rightarrow \mu^{+} \mu^{-}$proceed via CKM suppressed Z-penguin or W-box diagrams. Additionally, the decays are helicity suppressed by a factor $\left(m_{\mu} / M_{B}\right)^{2}$. The Standard Model branching fraction is computed to be $B R\left(B_{s}^{0} \rightarrow \mu^{+} \mu^{-}\right) \sim 3.3 \times 10^{-9}[6]$. The branching fraction for $B_{d}^{0} \rightarrow \mu^{+} \mu^{-}$is further suppressed by the CKM ratio $\left|V_{t d} / V_{t s}\right|^{2}$ leading to a predicted Standard Model branching fraction of $\sim 1 \times 10^{-10}$. These rare decays are very sensitive to any New Physics model with new scalar or pseudo-scalar interactions, in particular to models with an extended Higgs sector. In the MSSM the branching fractions are proportional to $\tan ^{6} \beta$. The current upper limit of the $B_{s}^{0} \rightarrow \mu^{+} \mu^{-}$branching fraction from the $\mathrm{CDF}$ and $\mathrm{D} \varnothing$ experiments is $4.7 \times 10^{-8}[3,7]$.

Experimentally, the search for the $B_{s} \rightarrow \mu^{+} \mu^{-}$decay has to deal with the problem of an enormous level of background, dominated by random combinations of two muons originating from two distinct $B$ decays. It can be kept under control by exploiting the excellent tracking and vertexing capabilities of the $\mathrm{LHC} b$ detector. Due to a very low muon misidentification rate $(0.5 \%)$, two-body hadronic decays, $B \rightarrow h^{+} h^{-}$, where the hadrons are misidentified as muons, do not contribute to a significant level. The $\mathrm{LHC} b$ experiment estimates the total efficiency to select $B_{s} \rightarrow \mu^{+} \mu^{-}$events to $10 \%$, including trigger efficiency. As shown in Figure 2, this yields to a sensitivity to exclude a branching ratio down to $7 \times 10^{-9}$ with a confidence level of $90 \%$, with $0.5 \mathrm{fb}^{-1}$ of data. A data-set of $2 \mathrm{fb}^{-1}\left(6 \mathrm{fb}^{-1}\right)$ will allow the observation of the Standard Model branching fraction with a $3 \sigma(5 \sigma)$ significance.

The ATLAS and CMS collaborations estimate their sensitivity to be able to exclude a branching ratio down to $10^{-8}$ at $90 \% \mathrm{CL}$ with a dataset of $2 \mathrm{fb}^{-1}[8]$.

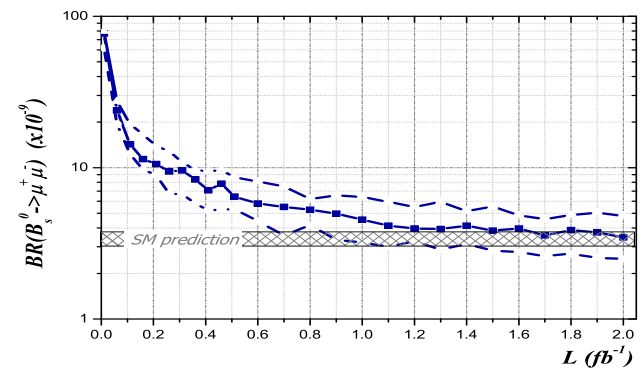

Figure 2: Expected upper limit for the branching ratio of the $B_{s} \rightarrow \mu^{+} \mu^{-}$decay at $90 \% \mathrm{CL}$ for $\mathrm{LHC} b$ as a function of the integrated luminosity. A prediction of the Standard Model is also indicated.

\section{$3.2 \quad b \rightarrow s \ell^{+} \ell^{-}$}

In the Standard Model, the electroweak penguin decays $b \rightarrow s \ell^{+} \ell^{-}$are only induced at loop level which leads to small branching fractions and thus a high sensitivity to contributions from physics beyond the Standard Model. The decay $B_{d} \rightarrow K^{\star} \mu^{+} \mu^{-}\left(\mathcal{B R} \sim 1.2 \times 10^{-6}\right)$ is experimentally best accessible due to the clear dimuon signature. An angular analysis of this decay is well suited to search for New Physics as most scenarios make definite predictions for the forward-backward asymmetry $\left(A_{F B}\right)$ of the $\mu^{+}$relative to the $B$ direction in the dimuon rest frame as a function of the dimuon mass. In particular $s_{0}$, the zero crossing point of $A_{F B}$, is predicted with small theoretical uncertainties and will provide a stringent test of the Standard Model and New Physics scenarios. 
The decay $B_{d} \rightarrow K^{\star} \mu^{+} \mu^{-}$can be triggered and reconstructed in LHCb with a total efficiency of $\sim 1 \%$ [9]. This results in an expectation of $\sim 7200$ signal events for an integrated luminosity of $2 \mathrm{fb}^{-1}$ with a background-to-signal ratio of about 0.5. A simple counting analysis results in a precision of $0.5 \mathrm{GeV}^{2}$ on the zero crossing point $s_{0}$. In addition to measuring $A_{F B}, \mathrm{LHC} b$ is able to extract other angular observables, such as the longitudinal $K^{\star}$ polarization $F_{L}\left(q^{2}\right)$ or the transverse asymmetry $A_{T}^{(2)}\left(q^{2}\right)[10]$.

\section{Measurement of the CKM angle $\gamma$}

The BABAR and BELLE experiments have performed first direct measurements of the CKM angle $\gamma$ using $B \rightarrow D K$ tree decays. Limited by statistics, the precision is still much below the indirect determination from CKM fits [11]. The measurement exploits the interference between the two tree decay amplitudes $B^{-} \rightarrow D^{0} K^{-}$and $B^{-} \rightarrow \bar{D}^{0} K^{-}$, as shown in Figure 3. The interference of the $D^{0}$ and $\bar{D}^{0}$ decays into the same final state allows the observation of the phase difference between the two amplitudes,

$$
\frac{\mathcal{A}\left(B^{-} \rightarrow D^{0} K^{-}\right)}{\mathcal{A}\left(B^{-} \rightarrow \bar{D}^{0} K^{-}\right)}=r_{B} e^{\delta_{B}} e^{-i \gamma}
$$

where $r_{B}$ is the relative magnitude of the two amplitudes and $\delta_{B}$ is the strong phase difference.

LHCb has investigated the expected sensitivity for $\gamma$ using the ADS, GLW and the GGSZ method for the time integrated decay rates [12]. The different methods result into a similar sensitivity. For a data-sample corresponding to $2 \mathrm{fb}^{-1}$, it is $10-13^{\circ}$, depending on the strong phase $\delta_{B}$, which can be simultaneously determined.

An alternative way to determine $\gamma$ is the measurement of the time dependent CP asymmetry of the decays $B_{s} \rightarrow D_{s}^{\mp} K^{ \pm}$. As the $B_{s}$ and the $\bar{B}_{s}$ can both decay to $D_{s}^{ \pm} K^{\mp}$, there exists an interference between amplitudes with and without a preceding oscillation which gives access to sum of $\gamma+\Phi_{s}$. The main issue of this analysis is to separate the decay $B_{s} \rightarrow D_{s}^{\mp} K^{ \pm}$from the very similar but $\sim 15$ times more frequent decay $B_{s} \rightarrow D_{s}^{\mp} \pi^{ \pm}$. Here, the excellent $\mathrm{LHCb}$ particle identification based on the two RICH counters allows a significant reduction of the background contamination. For a data-sample of $2 \mathrm{fb}^{-1}$, a signal yield of $6200 B_{s} \rightarrow D_{s}^{\mp} K^{ \pm}$events with a background-to-signal ratio of $\sim 0.7$ is expected.
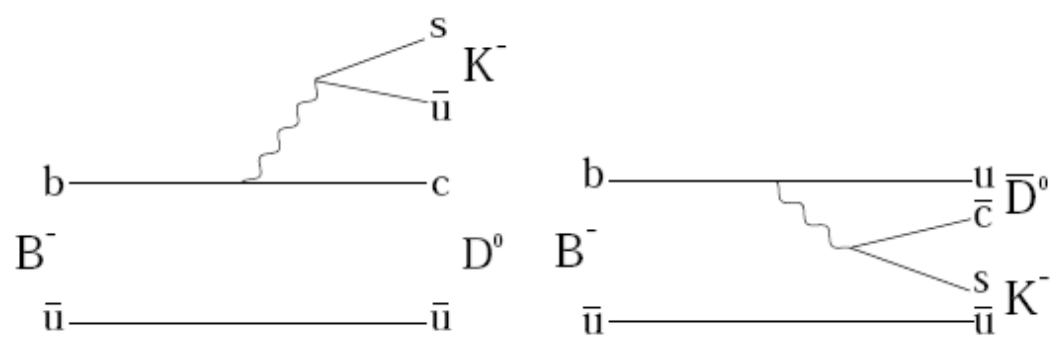

Figure 3: Feynman diagrams for the decays $B^{-} \rightarrow D^{0} K^{-}$and $B^{-} \rightarrow \bar{D}^{0} K^{-}$. 
The resulting sensitivity to $\gamma+\Phi_{s}$ varies between $9^{\circ}$ and $12^{\circ}$, dependent on $\delta_{B}$, the strong phase difference which can be determined from the data.

Table 1 summarizes the expected $\mathrm{LHC} b$ sensitivity, combining all tree-level measurements of $\gamma[13]$. A dataset corresponding to $0.5 \mathrm{fb}^{-1}$ would already allow the determination of $\gamma$ with an error below $10^{\circ}$.

\begin{tabular}{|c|c|}
\hline $\mathcal{L}_{\text {int }}$ & $\sigma_{\gamma}$ \\
\hline $0.5 \mathrm{fb}^{-1}$ & $7.8-10.1^{\circ}$ \\
$2 \mathrm{fb}^{-1}$ & $3.9-5.1^{\circ}$ \\
$10 \mathrm{fb} b^{-1}$ & $1.9-2.7^{\circ}$ \\
\hline
\end{tabular}

Table 1: Expected combined LHC $b$ sensitivity to measure $\gamma$, covering all values of $\delta_{B}$.

\section{Summary and outlook}

The large $b \bar{b}$ production cross section at the LHC provides a unique opportunity for the precision study of very rare $B$ decays. In particular the $B_{s}$ system could play a crucial role to identify new $C P$ violating effects originating from New Physics. Many of the observables constructed from rates, angular distributions, and $C P$ asymmetries for the loop suppressed $B$ decays show large sensitivities to New Physics contributions.

Already with a data-set of $0.5 \mathrm{fb}^{-1} \mathrm{LHC} b$ expects to perform sensitive tests of the Standard Model and New Physics scenarios beyond the current experiments: The $B_{s}$ mixing phase $\Phi_{s}$ can be measured with a precision of \pm 0.06 ; the reachable precision of the CKM phase $\gamma$ is estimated to be between $8^{\circ}$ and $10^{\circ}$; a $B_{s} \rightarrow \mu^{+} \mu^{-}$branching fraction of $5 \times 10^{-9}$ can be observed. These measurements, complemented by ATLAS and CMS, will either limit New Physics contributions to B decays or, more optimistically, uncover them.

\section{References}

[1] Slides: http: //indico. cern. $\mathrm{ch} /$ contributionDisplay $\cdot$ py? contribId=81\&sessionId=2\&conf Id=53294

[2] The CDF collaboration,Phys. Rev. Lett. 100, 16802 (2008), The D0 collaboration, hep-ex, arXiv:0802.2255v1, (2008).

[3] Heavy Flavor Averaging Group, http://www.slac.stanford.edu/xorg/hfag/

[4] The CDF collaboration, CDF/ANAL/BOTTOM/PUBLIC/9458 (2008).

[5] M. Calvi et al, CERN-LHCb-2009-025 (2009)

[6] M. Blanke, A.J. Buras, D. Guadagnoli and C. Tarantino JHEP0610, 003 (2006).

[7] The D0 Collaboration, http://wwwd0.fnal.gov/Run2Physics/WWW/results/bhtm (2006), The CDF Collaboration, CDF/ANAL/BOTTOM/PUBLIC/8176 (2006).

[8] G. Buchalla at al. B, D and $K$ decays, arXiv:0801.1833v1 [hep-ph] (2008)

[9] J. Dickens et al., CERN-LHCb-2007-038 (2007).

[10] U. Egede, CERN-LHCb-2007-057 (2007).

[11] CKM fitter: http://ckmfitter.in2p3.fr

[12] ADS: D. Atwood, I. Dunietz and A. Soni, Phys. Rev. Lett. 78, 3257 (1997), GLW: M. Gronau and D. London, Phys. Lett. B 253, 483 (1991), GGSZ: A. Giri, Yu. Grossman, A. Soffer and J. Zupan, Phys. Rev. D 68, 054018 (2003).

[13] K. Akiba et al., CERN-LHCb-2008-038 (2008). 